\title{
Mesiodens: presentación de tres casos
}

\author{
ML. Díaz Ortiz ${ }^{\mathrm{a}}$, M. Vicente Rodríguez ${ }^{\mathrm{b}}$

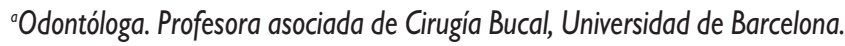 \\ Área Básica de Salud Garraf Rural. Sant Pere de Ribes, Barcelona. España. \\ 'Pediatra. Área Básica de Salud Garraf Rural. Sant Pere de Ribes, Barcelona. España.
}

\begin{abstract}
Resumen
El mesiodens es el diente supernumerario que aparece con mayor frecuencia en la dentición definitiva. En la mayoría de ocasiones se trata de un hallazgo casual. El diagnóstico precoz permite realizar la extracción de forma temprana, favoreciendo la erupción espontánea de los incisivos permanentes, y evita tener que efectuar otros tratamientos más complejos, quirúrgicos u ortodóncicos. Presentamos tres casos de pacientes pediátricos con mesiodens. Todos ellos fueron hallazgos radiológicos. En el primer caso, el paciente presentaba un mesiodens doble que impedía la erupción de los incisivos centrales y laterales superiores definitivos. En el segundo caso, el mesiodens provocó la rotación de los incisivos centrales superiores. Se le realizó una extracción temprana para favorecer la erupción espontánea de los incisivos. En el tercer caso, el mesiodens se hallaba en inclusión total, sin producir sintomatología ni complicaciones. Este hecho permitió la erupción normal del sector anterior. No ha requerido tratamiento y sólo se le realizan controles periódicos. Este paciente presentaba, además, agenesia de un premolar inferior. Es importante sospechar la presencia de un mesiodens en el paciente que acude a la consulta de pediatría u odontología cuando exista algún trastorno en la erupción de los incisivos superiores permanentes.
\end{abstract}

Palabras claves: Mesiodens. Diente supernumerario. Alteración de la erupción.

\section{Mesiodens: three case reports}

\section{Abstract}

Mesiodens is the supernumerary tooth that most frequently appears in the permanent dentition. In most cases this is an accidental finding. Early detection allows the early extraction. It also favors the spontaneous eruption of the permanent incisors and helps avoid more complex surgical or orthodontic treatments. We present three cases of paediatric patients with mesiodens, all of them have been radiological findings. In the first case, the patient had a double mesiodens which blocked the eruption of the upper permanent central and lateral incisors. In the second case, mesiodens has produced the rotation of maxillary central incisors. Early removal has been practiced in order to favor the spontaneous eruption of the incisors. In the third case, mesiodens is in total inclusion, not producing symptoms or complications, and it has allowed normal eruption of the previous sector. It has not required tre-

María Luisa Díaz Ortiz, Idiazo@ambitcp.catsalut.net

Los autores declaran no presentar conflictos de intereses en relación con la preparación y publicación de este artículo. 
atment, only periodic controls. This patient also presents agenesis of a lower premolar. It is reasonable to suspect the presence of a mesiodens in the patient who goes to pediatric or odontology consultation when there is some kind of disorder in the eruption of the upper permanent incisors.

Key words: Mesiodens. Supernumerary teeth. Eruption disturbance.

\section{Introducción}

Un mesiodens es un diente supernumerario localizado en la región de los incisivos centrales superiores. Es el diente supernumerario más común. Representa el $50-80 \%$ del total de ellos. Puede presentarse como único o múltiple en un mismo individuo, así como coincidir con otros dientes supernumerarios o con agenesia de otros dientes. La frecuencia varía entre $0,15-2,65 \%$ y predomina en el sexo masculino. El 80-90\% aparece en el maxilar superior. La situación más habitual es que se encuentre incluido completamente y sólo esté erupcionado en un $25 \%$ de los casos ${ }^{1-2}$.

La inclusión constituye un obstáculo mecánico y puede ocasionar persistencia del diente temporal. En los incisivos superiores permanentes puede provocar diastema interincisal, inclusión, malposición, lesiones periodontales y radiculares. Otras complicaciones descritas son la erupción en la cavidad nasal y la formación de quistes foliculares ${ }^{3-5}$.

Debemos sospechar la presencia de un mesiodens incluido en el caso de que exista una asimetría en la forma de erupcionar los incisivos superiores, si existe retraso en la exfoliación de los incisivos temporales -sobre todo, si el retraso es asimétrico-, también ante la erupción ectópica de uno o ambos incisivos y cuando se produzca la presencia de un diastema interincisal. En ocasiones producen una protuberancia en las mucosas vestibular o palatina que se pueden palpar. La mayoría son asintomáticos y el estudio radiográfico -mediante la ortopantomografía, la radiografía periapical y la oclusal maxilar- nos dará el diagnóstico ${ }^{1,2,5,6}$.

El tratamiento del mesiodens erupcionado debe ser la extracción por motivos estéticos y funcionales, ya que suelen ser displásicos, están en malposición y ocupan un espacio en la arcada'. En el caso de que esté incluido, la extracción será el tratamiento de elección. Sin embargo, si no produce sintomatología o existe un elevado riesgo de lesionar el diente permanente, se aconseja la abstención terapéutica y realizar controles clínicos y radiológicos periódicos ${ }^{3}$.

Presentamos tres casos clínicos que acuden a la consulta odontológica del 
Centro de Atención Primaria de Garraf Rural (Barcelona). La mayoría de los niños visitados en la consulta de odontología fueron derivados desde el servicio de pediatría, pues es aquí donde se observan en primer lugar las alteraciones dentales en los niños.

\section{Casos clínicos}

\section{Caso n. ${ }^{\circ} 1$}

Paciente varón de 6 años, sin antecedentes patológicos de interés, que acude para una revisión a la consulta de odontología. En la exploración intraoral presentaba una exfoliación de los cuatro in- cisivos superiores sin erupcionar ninguno de los definitivos. En la palpación se apreciaba una tumefacción en el cortical vestibular que correspondía a las coronas de los incisivos centrales superiores, pero éstos estaban localizados muy separados de la línea media. Se solicitó una ortopantomografía para valorar la posición y el estado de la erupción de los incisivos superiores. La radiografía mostraba la presencia de dos mesiodens en la línea media (figura 1). El del lado derecho se encontraba en posición más apical y era de mayor tamaño que el izquierdo. Ambos se hallaban en posición vertical y presentaban una forma conoide. Provo-

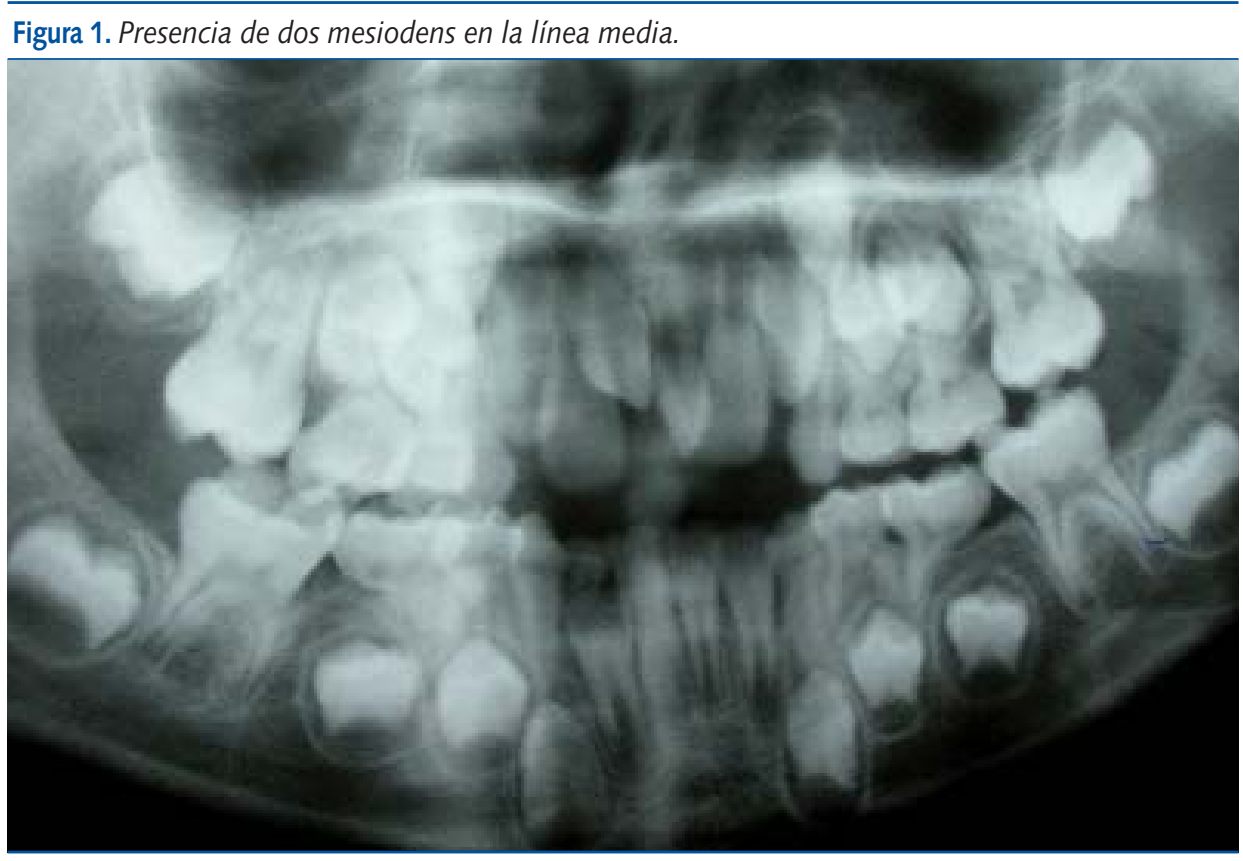


caban un gran diastema interincisal e impedían la correcta erupción de los incisivos centrales superiores. Se informó a la madre de la necesidad de realizar la extracción de los dientes supernumerarios para la correcta erupción dentaria del sector anterior, y el paciente fue derivado al servicio de cirugía maxilofacial para la extracción quirúrgica de los mesiodens. Antes de la cirugía, el paciente presentaba la erupción de 1/3 de la corona del incisivo superior izquierdo y la cúspide del mesiodens izquierdo. El servicio de cirugía maxilofacial decide realizar la intervención en dos fases: primero hace la exodoncia quirúrgica del mesiodens iz- quierdo y realizará la del derecho cuando éste tenga una posición más baja. El paciente precisará controles periódicos para controlar la erupción dentaria y valorar la necesidad de un tratamiento ortodóncico posterior para cerrar el diastema interincisal y permitir, además, la erupción de los incisivos laterales superiores.

\section{Caso n. ${ }^{\circ} 2$}

Paciente varón de 5 años, sin antecedentes médicos de interés, que acude a la consulta de odontología por presentar una fístula en la mucosa vestibular en el incisivo central superior derecho. El padre refiere que a los 2 años de edad tuvo un

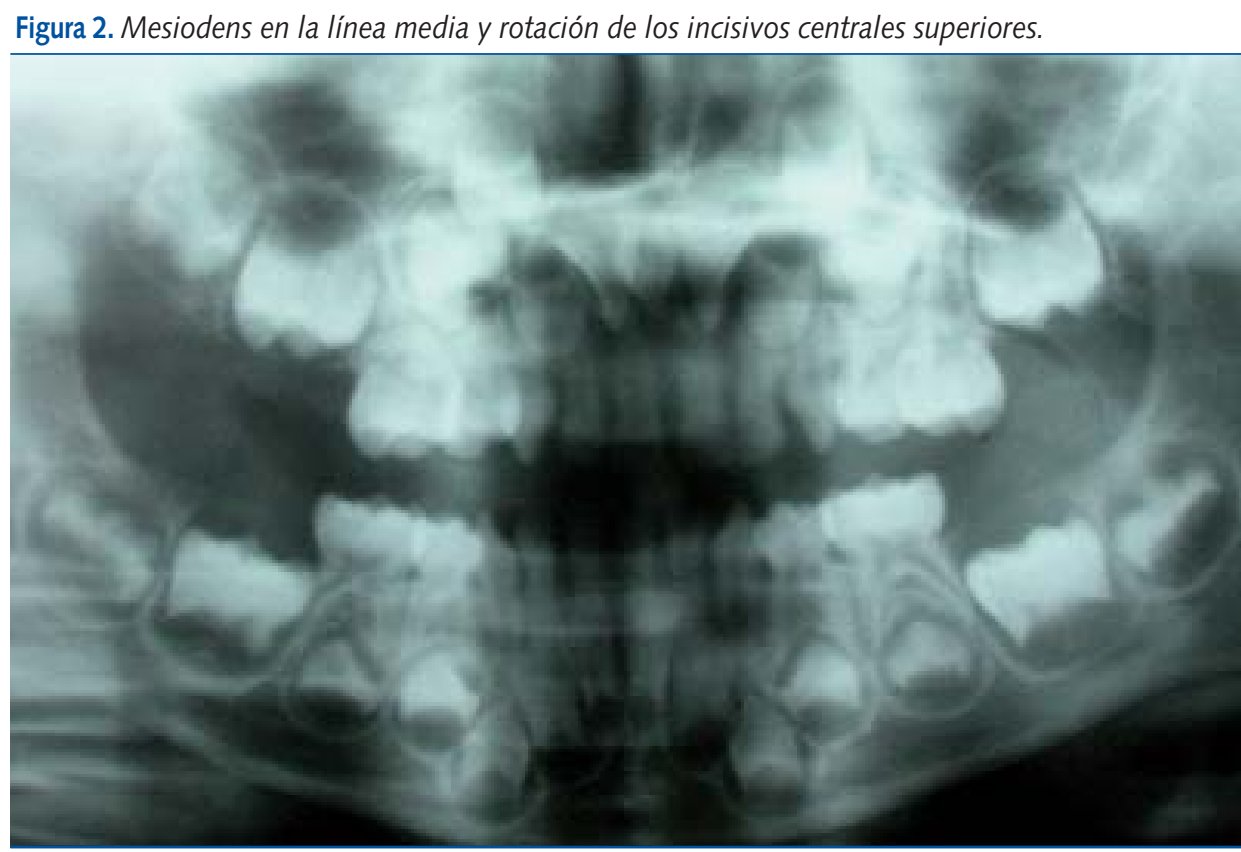


traumatismo en los incisivos superiores y desde entonces no había tenido ninguna sintomatología asociada. En la exploración dental observamos la presencia de toda la dentición temporal. Se le prescribió tratamiento antibiótico durante una semana y se le solicitó una ortopantomografía para valorar el estado de los incisivos. En la radiografía (figura 2 ) vimos la presencia de un mesiodens en la línea media, de forma conoide y sin formación completa de la raíz. Su cúspide se localizaba a nivel del 1/3 apical de los incisivos superiores temporales. Los dos incisivos centrales superiores definitivos sólo tenían formada la corona. Ambos se encontraban rotados, con las caras vestibulares hacia la línea media y las caras palatinas hacia los incisivos laterales. Los gérmenes de los incisivos laterales estaban bien posicionados, pero situados ligeramente más bajos que los centrales. Se recomienda que le realicen la pulpotomía del incisivo central superior derecho temporal. Se decide esperar a que los incisivos centrales definitivos estén más formados y en posición más favorable antes de extraer el supernumerario. A los 6 años y medio le realizan la exodoncia quirúrgica del mesiodens y de los dos incisivos centrales temporales. A los 7 años todavía no ha erupcionado ninguno de los incisivos superiores, pero en el estudio radiológico se observa que éstos tienen una posición más favorable para su erupción.

\section{Caso n. ${ }^{\circ} 3$}

Paciente de 13 años, sin antecedentes patológicos de interés, que viene a la consulta para una revisión odontológica. A los 10 años tuvo que acudir por no exfoliarse el segundo molar temporal izquierdo. Le realizaron una ortopantomografía y observaron que presentaba agenesia del segundo premolar inferior izquierdo y un mesiodens en la arcada superior. Le recomendaron realizarse controles periódicos. En la exploración bucodental de los 13 años se observa la persistencia del molar temporal. El sector anterior está erupcionado correctamente. El mesiodens no ha provocado ninguna anomalía en la posición de los incisivos superiores. En la ortopantomografía se aprecia el mesiodens incluido en una ligera distoversión, con la cúspide localizada entre las raíces del incisivo central y el incisivo lateral superior izquierdo, a nivel del 1/3 medio radicular, y el ápice en la línea media (figura 3). A los 16 años el paciente acude por presentar una caries en el molar temporal. Solicitamos una nueva radiografía para valorar si se puede obturar el molar, controlar el supernumerario y valorar los cordales. En la ortopantomografía observamos el mesiodens 
Figura 3. Mesiodens y agenesia del segundo premolar inferior izquierdo a los 13 años.

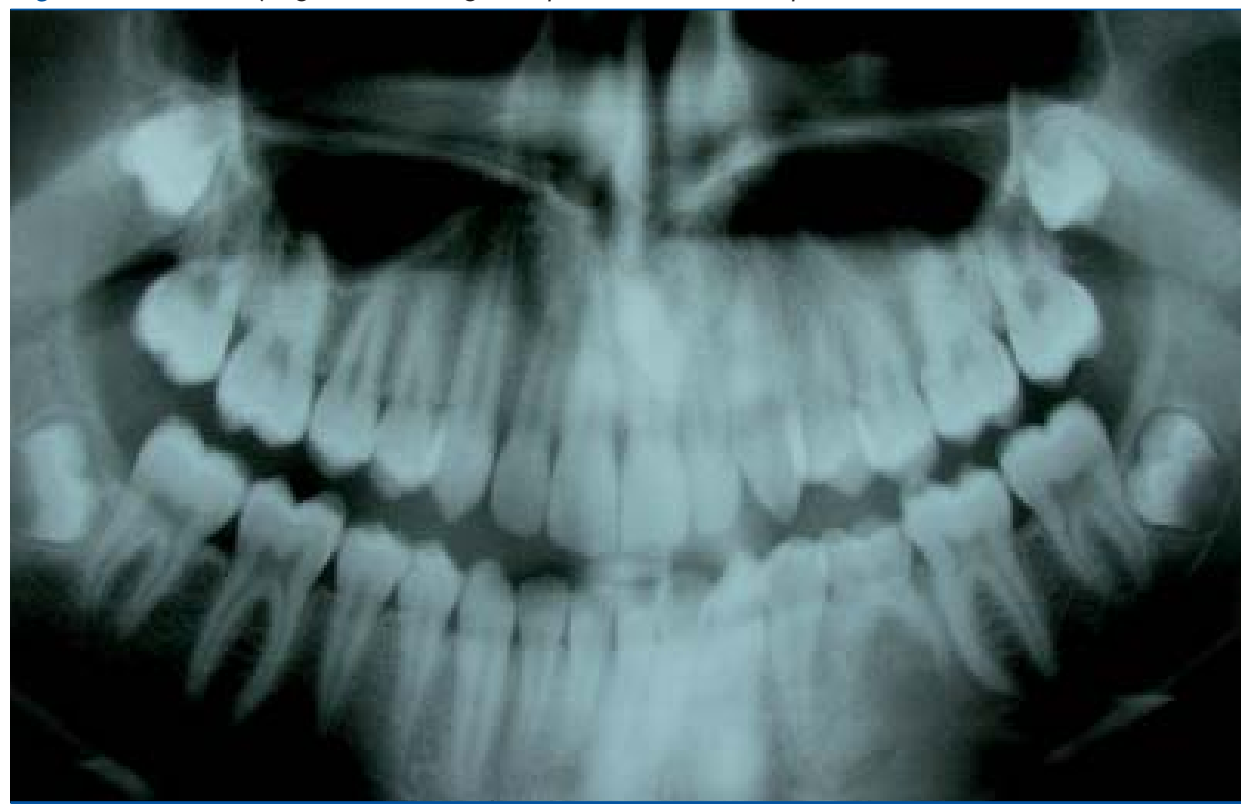

en la línea media, ligeramente más verticalizado, pero sin cambios significativos en su posición respecto a las radiografías anteriores. Decidimos seguir realizando controles periódicos del supernumerario y lo remitimos a cirugía maxilofacial para la exodoncia de los cordales inferiores por presentar falta de espacio para su erupción. Recomendamos la obturación del molar temporal.

\section{Discusión}

En la consulta de odontología de nuestro centro de asistencia primaria hemos registrado, desde el año 2007, 6 casos con presencia de mesiodens. Destacamos los casos 1 y 2 presentados por ser poco frecuentes en pacientes sin enfermedades ni síndromes asociados; el primero de ellos por ser un mesiodens doble. Aunque lo más frecuente es la presencia de uno solo, hay autores como Hernández y cols. ${ }^{7}$ que también presentan casos de mesiodens dobles en niños. Gündüz et al. ${ }^{5}$ recogen los casos de 69 pacientes con mesiodens -53 presentaban un mesiodens $(76,8 \%)$ y 16 , dos $(23,1 \%)-$. Salcido y cols. ${ }^{8}$ hallaron 36 casos con mesiodens: 31 únicos $(86,1 \%)$ y 5 dobles $(13,9 \%)$. Ferres Padró y cols. ${ }^{9}$ hallaron en 42 casos con mesiodens: 36 únicos $(45,6 \%), 4$ dobles $(5,1 \%)$ y 2 más de tres 
mesiodens (2,5\%). El tercer caso también es peculiar por coincidir la presencia de un supernumerario con la agenesia de un premolar.

Los estudios sobre dientes supernumerarios suelen mostrar que el mesiodens es el que aparece con mayor frecuencia, seguido por los premolares y los distomolares $^{3,8}$. Fernández y cols. ${ }^{3}$ realizaron la extracción de 145 supernumerarios en 102 pacientes. El mesiodens fue el más frecuente: el $46,9 \%$ de los pacientes lo presentaban. También lo fue para Salcido y cols. ${ }^{8}$, quienes revisaron a 2.245 pacientes y hallaron 102 dientes supernumerarios en 72 pacientes, 36 de los cuales lo presentaron. Ferrés Padró y cols. ${ }^{9}$ realizaron la exodoncia de 113 supernumerarios no erupcionados en 79 pacientes pediátricos. El mesiodens fue el diente supernumerario más frecuente $(53,2 \%$ de los pacientes), con una cantidad total de 42.

En otros estudios, como el de Leco Berrocal y cols. ${ }^{4}$, los distomolares son los más habituales, seguidos de los mesiodens y los premolares. En una población de 2.000 pacientes, hallaron un total de 24 dientes supernumerarios en 21 pacientes, de los cuales el 42,8\% fueron distomolares y el $28,6 \%$ mesiodens.

En muchas ocasiones, el diagnóstico se hace mediante el estudio radiológico, como ha ocurrido en los tres casos presentados. La ortopantomografía realizada como visita de control nos avisa de la presencia de dientes supernumerarios sin existir signos clínicos, ya que en un alto porcentaje son asintomáticos ${ }^{1,2,6}$. Estudios como los de Fernández y cols. ${ }^{3}$ y Gündüz et al. ${ }^{5}$, que extrajeron 68 y 85 mesiodens respectivamente, muestran que en el $39,7 \%$ de los casos del primer estudio y en el $22,3 \%$ del segundo habían sido asintomáticos.

En otras ocasiones provocan complicaciones. Fernández y cols. ${ }^{3}$ hallaron que el $51,5 \%$ de los 68 mesiodens extraídos había producido inclusión de los dientes permanentes, el 7,4\% malposición dentaria y diastema, y en un caso formación de un quiste folicular. Gündüz et al. ${ }^{5} \mathrm{se}$ ñalaron que en el $38,8 \%$ de los 85 mesiodens extraídos se produjo retraso en la erupción de los incisivos permanentes, en el $17,6 \%$ diastema interincisal, en el $16,4 \%$ alteración en la posición de los incisivos permanentes erupcionados y en el 4,7\% reabsorción de los dientes adyacentes. De los dientes supernumerarios, los mesiodens suelen ser los que presentan más complicaciones, seguidos de los premolares; son menos frecuentes en los caninos y los distomolares 4 .

Además de la ortopantomografía, en algunos casos debemos completar el es- 
tudio radiológico con una radiografía periapical, oclusal, lateral de cráneo o tomografía computarizada para determinar la localización exacta y la relación con estructuras adyacentes, antes de realizar su extracción ${ }^{2,6}$.

En la dentición primaria normalmente no está indicada la extracción del mesiodens por el alto riesgo de desplazar o dañar el desarrollo de los incisivos permanentes. En la dentición mixta, existen dos tendencias terapéuticas respecto al momento más indicado de realizar la extracción: la extracción temprana (antes de la formación radicular de los incisivos permanentes) y la tardía (después de completar la formación radicular) ${ }^{2,3}$. En los casos 1 y 2 se ha optado por la temprana para favorecer la erupción espontánea de los incisivos, evitar complicaciones mecánicas y minimizar la necesidad de un tratamiento ortodóncico y/o quirúrgico posterior más complejo. En el caso 3 se ha optado por no extraerlo por no producir sintomatología, pero precisará controles clínicos y radiológicos periódicos.

Es importante el trabajo en equipo dentro de los centros de asistencia primaria. El servicio de pediatría juega un papel relevante en la motivación de los padres para que los niños acudan desde una edad temprana a las revisiones odontológicas. Hemos de tener en cuenta que acuden más frecuentemente a las consultas de pediatría que a la del odontólogo.

Concluimos haciendo hincapié en la importancia de diagnosticar de forma precoz la presencia de un mesiodens en el niño desde las consultas de Odontología y/o Pediatría de los centros de Atención Primaria. Su extracción de forma temprana permitirá, en la mayoría de los casos, la erupción espontánea de los incisivos afectados, evitando otras complicaciones asociadas y tratamientos posteriores más complejos.

\section{Bibliografía}

1. Gay Escoda C, Mateos Micas M, España Tost A, Gargallo Albiol J. Otras inclusiones dentarias. Mesiodens y otros dientes supernumerarios. Dientes temporales supernumerarios. Dientes temporales incluidos. En: Gay Escoda C, Aytés Berini $L$, editores. Tratado de Cirugía Bucal. Tomo I. 1. a ed. Madrid: Ergon; 2004. p. 497534.

2. Russell KA, Folwarczna MA. Mesiodens: diagnosis and management of a common supernumerary tooth. J Can Dent Assoc. 2003;69: 362-6.

3. Fernández Montenegro P, Valmaseda Castellón E, Berini Aytés L, Gay Escoda C. Estudio retrospectivo de 145 dientes supernumerarios. Med Oral Patol Oral Cir Bucal. 2006;11:240-5.

4. Leco Berrocal MI, Martín Morales JF, Martínez González JM. Estudio observacional sobre la 
frecuencia de dientes supernumerarios en una población de 2.000 pacientes. Med Oral Patol Oral Cir Bucal. 2007;12:96-100.

5. Gündüz K, Celenk P, Zengin Z, Sümer P. Mesiodens: a radiographic study in children. J Oral Sci. 2008;50:287-91.

6. Cahuana Cárdenas A, Alfaro A, Pérez B, Coelho $A$. Dientes supernumerarios anteriores no erupcionados. Revisión de 125 casos. RCOE. 2003;8:263-71.

7. Hernández $M$, Ferreira LP. Mesiodens múltiples sin antecedentes sindrómicos. A propósito de dos casos. J Am Dent Assoc (ed. esp.). 2009;4: 140-4.

8. Salcido García JF, Ledesma Montes C, Hernández Flores $F$, Pérez $D$, Garcés Ortiz $M$. Frecuencia de dientes supernumerarios en una población mexicana. Med Oral Patol Oral Cir Bucal. 2004;9:403-9.

9. Ferrés Padró E, Prats Armengol J, Ferrés Amat $E$. A descriptive study of 113 unerupted supernumerary teeth in 79 pediatric patients in Barcelona. Med Oral Patol Oral Cir Bucal. 2009;14: 146-52. 